\title{
Correlação clínico-radiográfica das fraturas articulares completas do rádio distal tratadas cirurgicamente
}

Clinical-radiographic correlation of complete articular fractures of the distal radius treated surgically Correlación clínico-radiográfica de fracturas articulares completas de radio distal tratadas quirúrgicamente

Thiago Agostini Pereira ALBENY ${ }^{1}$

Breno Alves de Souza VAZ ${ }^{2}$

Luis Guilherme Rosifini Alves REZENDE ${ }^{3}$

Filipe Jun SHIMAOKA ${ }^{3}$

Amanda Favaro CAGNOLATI ${ }^{3}$

Alex Calderon IRUSTA ${ }^{3}$

Luiz Garcia MANDARANO-FILHO ${ }^{3}$

Nilton MAZZER ${ }^{4}$

${ }^{1}$ Cirurgião de Mão no Hospital Sírio Libanês (Brasília), formado no programa de Cirurgia da Mão, Hospital das Clínicas, Faculdade de Medicina de Ribeirão Preto Universidade de São Paulo (HC-FMRP-USP), Ribeirão Preto, SP, Brasil

${ }^{2}$ Residente no programa de Cirurgia da Mão, Hospital das Clínicas, Faculdade de Medicina de Ribeirão Preto, Universidade de São Paulo (HC-FMRP-USP), Ribeirão Preto, SP, Brasil

${ }^{3}$ Médico Assistente e Preceptor do Programa de Cirurgia da Mão, Hospital das Clínicas, Faculdade de Medicina de Ribeirão Preto, Universidade de São Paulo (HC-FMRP-USP), Ribeirão Preto, SP, Brasil

${ }^{4}$ Professor Titular e Chefe da Divisão de Cirurgia da Mão, Hospital das Clínicas, Faculdade de Medicina de Ribeirão Preto, Universidade de São Paulo (HC-FMRP-USP), Ribeirão Preto, SP, Brasil

\section{Resumo}

Introdução: As fraturas da extremidade distal do rádio representam uma das fraturas mais comuns. Diversos fatores descritos na literatura influenciam nos seus resultados após manejo cirúrgico; como a fragmentação articular, a restauração cirúrgica da anatomia do rádio, a reabilitação pós-operatória, entre outros. Objetivo: analisar o resultado funcional dos pacientes operados de fraturas articulares completas da extremidade distal do rádio e correlacionar estes resultados com os parâmetros radiológicos comumente utilizados. Métodos: 18 pacientes entre 18 a 65 anos, submetidos ao tratamento cirúrgico das fraturas do rádio distal do tipo AO 23C, no período de janeiro de 2014 a julho de 2016. Os seguintes parâmetros clínicos e radiográficos foram avaliados e submetidos a análise estatística: ADM (amplitude de movimento) do punho e antebraço, força de pinças e de preensão, PRWE (Patient Rated Wrist Evaluation), classificação AO da fratura e parâmetros radiográficos pós-operatórios. Resultados: A análise de regressão linear mostrou correlação estatisticamente significativa considerando a inclinação radial e o desvio ulnar. Conclusão: Correlação estatisticamente significativa entre os parâmetros radiográficos e resultados funcionais é de difícil determinação. Novos estudos com maior amostragem e que correlacionem os parâmetros radiográficos e idade são necessários para melhor estudo do tema abordado.

Descritores: Fraturas do Rádio; Traumatismos do Punho; Fraturas Intra-Articulares.

\section{Abstract}

Introduction: Fractures of the distal end of the radius represent one of the most common fractures. Several factors described in the literature influence its results after surgical management; such as joint fragmentation, surgical restoration of the radius anatomy, postoperative rehabilitation, among others. Objective: to analyze the functional outcome of patients operated on complete joint fractures of the distal end of the radius and to correlate these results with the radiological parameters commonly used. Methods: 18 patients aged 18 to 65 years, who underwent surgical treatment for AO 23C distal radius fractures, from January 2014 to July 2016. The following clinical and radiographic parameters were evaluated and submitted to statistical analysis: ADM (range of motion) of the wrist and forearm, forceps and grips, PRWE (Patient Rated Wrist Evaluation), AO classification of the fracture and postoperative radiographic parameters. Results: The linear regression analysis showed a statistically significant correlation considering the radial inclination and the ulnar deviation. Conclusion: Statistically significant correlation between radiographic parameters and functional results is difficult to determine. New studies with larger sampling and that correlate radiographic parameters and age are necessary for a better study of the topic addressed.

Descriptors: Radius Fractures; Wrist Injuries; Intra-Articular Fractures.

\section{Resumen}

Introducción: Las fracturas del extremo distal del radio representan una de las fracturas más frecuentes. Varios factores descritos en la literatura influyen en sus resultados tras el manejo quirúrgico; tales como fragmentación articular, restauración quirúrgica de la anatomía del radio, rehabilitación postoperatoria, entre otros. Objetivo: analizar los resultados funcionales de los pacientes operados de fracturas articulares completas del extremo distal del radio y correlacionar estos resultados con los parámetros radiológicos comúnmente utilizados. Métodos: 18 pacientes de 18 a 65 años, que fueron sometidos a tratamiento quirúrgico por fracturas de radio distal AO 23C, desde enero de 2014 a julio de 2016. Se evaluaron y sometieron a análisis estadístico los siguientes parámetros clínicos y radiográficos: ADM (rango de movimiento) de la muñeca y el antebrazo, fuerza de sujeción y agarre, PRWE (Evaluación de muñeca clasificada por el paciente), clasificación AO de la fractura y parámetros radiográficos posoperatorios Resultados: El análisis de regresión lineal mostró una correlación estadísticamente significativa considerando la inclinación radial y la desviación cubital. Conclusión: Es difícil determinar una correlación estadísticamente significativa entre los parámetros radiográficos y los resultados funcionales. Nuevos estudios con mayor muestreo y que correlacionen parámetros radiográficos y edad son necesarios para un mejor estudio del tema abordado.

Descriptores: Fracturas del Radio; Traumatismos de la Muñeca; Fracturas Intraarticulares.

\section{INTRODUÇÃO}

A fratura da extremidade distal do rádio é a fratura mais comum do adulto, correspondendo a aproximadamente $10-17,5 \%{ }^{1,2}$. Apresenta incidência bimodal, na qual os indivíduos do sexo masculino são mais acometidos entre os mais jovens (por volta dos 40 anos); enquanto as mulheres são as mais acometidas por volta dos 60 anos. O mecanismo de trauma mais frequente é a queda da própria altura (66-77\%); responsável principalmente pelas fraturas extra-articulares nas mulheres. As fraturas intraarticulares representam 25 a $35 \%$ das fraturas, estão associadas a trauma de alta energia e afetam mais os indivíduos do sexo masculino ${ }^{3-8}$.

O diagnóstico é realizado pela anamnese e exame clínico, associados a radiografias do punho em duas incidências (póstero-anterior e perfil). Em suspeita ou vigência de acometimento articular, podem-se adicionar as incidências oblíquas e a 
tomografia computadorizada para melhor estudo da fratura e conduta terapêutica ${ }^{4,9,10}$.

Diferentes classificações foram propostas para as fraturas da extremidade articular do rádio, como as propostas pela AO Foundation (Arbeitsgemeinschaft für Osteosynthesefragen Foundation), Fernandez, Universal, Frykman, Melone, entre outras ${ }^{2,4,11,12}$. As fraturas articulares completas do rádio distal são classificadas pela $\mathrm{AO}$ como tipo $23 \mathrm{C}$, sendo subdivididas em C1, C2 e C3 considerando o padrão da fragmentação óssea ${ }^{2,13}$.

O tratamento destas fraturas pode ser conservador ou cirúrgico, levando-se em consideração a idade, condição clínica do paciente e de partes moles, presença de lesões associadas, critérios radiográficos da fratura e sua classificação $^{2,4,14-16}$. Critérios clínicos e radiológicos capazes de direcionar o tratamento foram apontados na literatura, principalmente o acometimento articular. Grande parte dos autores consideram a presença de cominuição articular e/ou fragmento articular com desvio maior que $1 \mathrm{~mm}$ como indicações de tratamento cirúrgico ${ }^{2,17-19}$.

Apesar do avanço tecnológico dos implantes e das técnicas de fixação, os resultados do tratamento das fraturas da extremidade articular do rádio são variáveis e dependentes de múltiplos fatores ${ }^{12,15,19-21}$.

$\mathrm{O}$ acometimento articular e o grau de cominuição, além da restauração da superfície articular e da altura radial, são considerados alguns desses fatores, bem como comorbidades do paciente, reabilitação, entre outros ${ }^{2,22-26}$. A avaliação funcional destes pacientes é analisada através da amplitude de movimento do punho, a força de preensão e de pinça; e traduzidos através de escores funcionais, como o PRWE (Patient Rated Wrist Evaluation) ${ }^{27,28}$.

O objetivo deste estudo é analisar o resultado funcional dos pacientes com fraturas articulares completas da extremidade distal do rádio submetidos ao tratamento cirúrgico e correlacionar estes resultados com os parâmetros radiológicos comumente utilizados.

\section{MATERIAL E MÉTODO}

Trata-se de estudo retrospectivo realizado no Hospital das Clínicas da Faculdade de Medicina de Ribeirão Preto da Universidade de São Paulo (USP) em Ribeirão Preto (SP), Brasil, e aprovado pelo Comitê de Ética em Pesquisa do Hospital das Clínicas da Faculdade de Medicina de Ribeirão Preto (CAAE: 74766117.1.0000.5440). Os critérios de inclusão foram pacientes com idade entre 18 a 65 anos com fratura do rádio distal do tipo $\mathrm{AO} 23 \mathrm{C}$ submetidos ao tratamento cirúrgico no período entre janeiro de 2014 a julho de 2016 que completaram 24 meses do período pós-operatório. A abordagem foi realizada através do uso de placas volares bloqueadas de ângulo-fixo, associadas ou não a placas radiais e/ou dorsais. Os critérios de exclusão foram pacientes com qualquer lesão prévia ou concomitante nos membros superiores e pacientes que não completaram o tempo mínimo de seguimento pósoperatório. Os seguintes parâmetros clínicos e radiográficos foram avaliados: 1) ADM (amplitude de movimento) do punho e antebraço medido através de goniômetro; comparado ao membro superior contralateral; 2) força de pinça digital polpa-polpa e pinça trípode medidas através de dinamômetro apropriado; comparado ao membro superior contralateral; 3) força de preensão medida através de dinamômetro Jamar, comparado ao membro superior contralateral; 4) PRWE, questionário baseado em 15 itens (5 itens relacionados a dor, 6 itens relacionados a atividades específicas e 4 relacionados a atividades do dia a dia), com cada item sendo pontuado de 0 a 10. A soma de todos os itens pode variar de 0 (melhor resultado) a 150 (pior resultado) ${ }^{28}$; 5) Classificação AO da fratura pela radiografia e tomografia pré-operatórias ${ }^{13}$; 6) Radiografia pósoperatória (degrau e desvio articular, altura radial, inclinação radial e volar).

Os dados coletados foram inicialmente apresentados de forma descritiva. A correlação entre os dados clínicos (ADM, força das pinças, força de preensão, PRWE) foram correlacionados com os dados radiográficos (degrau e desvio articular, altura radial, inclinação radial e volar) através do coeficiente de correlação de Spearman, onde valores próximos a 1 ou -1 denotam uma forte correlação entre as variáveis analisadas. Para efeito estatístico foi considerado o nível de confiança de $\mathrm{p}<0,05$. Nos casos onde $\mathrm{p}<0,05$, foram construídos gráficos de regressão linear para ilustrar a correlação entre as duas variáveis. Em todos os testes estatísticos foi utilizado o software R -versão. 3.0.1.

\section{RESULTADOS}

Foram selecionados 28 pacientes, dos quais, 10 não puderam completar o seguimento pósoperatório devido mudança de cidade ou não comparecimento aos retornos. Ao final, 18 pacientes foram avaliados e incluídos no estudo.

Entre os pacientes avaliados, 11 eram do sexo masculino e 7 do sexo feminino, com média de idade de 41,44 anos (mediana:44; desvio padrão 12,87). As fraturas foram classificadas de acordo com a classificação AO 23C $(\mathrm{C} 1: \mathrm{n}=2 ; \mathrm{C} 2$ : $\mathrm{n}=2 ; \mathrm{C} 3$ : $\mathrm{n}=14)$. A Tabela 1 ilustra os valores médios encontrados referentes a ADM expresso em graus, comparando o lado afetado ao não afetado. A Tabela 2 apresenta os valores médios encontrados referentes aos exames de pinça e preensão, comparando o lado afetado e o não afetado.

Os valores médios e o desvio padrão dos parâmetros da avaliação radiográfica encontram-se disponíveis na tabela 3. Comparado ao lado 
contralateral, a perda média de altura radial foi de $0,72 \mathrm{~mm}$ ( $94 \%$ do valor do lado contralateral); de inclinação radial foi de $2,63^{\circ}$ (88\% do valor do lado contralateral); e de inclinação volar foi de $8,47^{\circ}$ (23\% do valor do lado contralateral). Os dados referentes aos parâmetros radiográficos foram correlacionados um a um com os dados clínicos obtidos previamente. Para que a correlação fosse válida, os valores do coeficiente de correlação de Spearman encontrados deveriam estar o mais próximo de 1 ou -1 e com valor de $\mathrm{p}<0,05$.

Tabela 1. Média \pm DP dos obtidos durante a avaliação da ADM Goniométrica em graus

\begin{tabular}{l|c|c|c|}
\hline & $\begin{array}{c}\text { Lado afetado } \\
(\boldsymbol{n = 1 8})\end{array}$ & $\begin{array}{c}\text { Lado não } \\
\text { afetado (n=18) }\end{array}$ & Diferença \\
\hline Flexão de punho & $50,55 \pm 14,08$ & $71,66 \pm 10,43$ & $21,11 \pm 14,29$ \\
\hline Extensão de punho & $44,55 \pm 14,81$ & $57,5 \pm 14,87$ & $12,94 \pm 15,79$ \\
\hline Desvio Radial & $17,05 \pm 5,85$ & $21,94 \pm 5,46$ & $4,88 \pm 5,65$ \\
\hline Desvio Ulnar & $41,94 \pm 9,57$ & $54,72 \pm 6,74$ & $4,88 \pm 5,65$ \\
\hline Supinação & $80,55 \pm 11,09$ & $85 \pm 9,85$ & $4,44 \pm 8,55$ \\
\hline Pronação & $71,94 \pm 21,63$ & $80 \pm 10,71$ & $8,05 \pm 17,66$ \\
\hline Fonte: Dados da Pesquisa & & &
\end{tabular}

Tabela 2. Média \pm DP dos dados obtidos durante os exames de pinça e preensão em kgf/N

\begin{tabular}{l|c|c|c}
\hline & $\begin{array}{c}\text { Lado afetado } \\
(\boldsymbol{n = 1 8})\end{array}$ & $\begin{array}{c}\text { Lado não } \\
\text { afetado }(\boldsymbol{n}=\mathbf{1 8})\end{array}$ & Diferença \\
\hline Pinça polpa-polpa & $\mathbf{1 1 , 2 2 \pm 5 , 3 5}$ & $\mathbf{1 4 , 4 4 \pm 5 , 0 0}$ & $3,22 \pm 3,43$ \\
\hline Pinça trípode & $12,33 \pm 6,03$ & $15,77 \pm 4,54$ & $3,44 \pm 2,95$ \\
\hline Chave & $16,88 \pm 7,02$ & $22,00 \pm 5,09$ & $5,11 \pm 5,31$ \\
\hline Preensão & $24,83 \pm 12,14$ & $34,44 \pm 11,61$ & $9,61 \pm 10,69$ \\
\hline Fonte: Dados da Pesquisa & &
\end{tabular}

Tabela 3. Média \pm DP dos dados obtidos durante a avaliação dos parâmetros radiográficos

\begin{tabular}{l|c|c}
\hline & $\begin{array}{c}\text { Lado afetado } \\
(\mathbf{n = 1 8})\end{array}$ & $\begin{array}{c}\text { Lado não- } \\
\text { afetado (n=18) }\end{array}$ \\
\hline Degrau articular (mm) & $0,11 \pm 0,47$ & 0,00 \\
\hline Desvio articular (mm) & $0,36 \pm 0,87$ & 0,00 \\
\hline Altura radial (mm) & $11,72 \pm 2,03$ & $12,44 \pm 1,92$ \\
\hline Inclinação radial (graus) & $20,41 \pm 2,47$ & $23,04 \pm 1,19$ \\
\hline Inclinação volar (graus) & $8,47 \pm 2,27$ & $11,81 \pm 2,11$ \\
\hline Fonte: Dados da Pesquisa & \multicolumn{2}{|c}{}
\end{tabular}

As Tabelas 4, 5 e 6 apresentam as correlações encontradas para a altura radial, inclinação radial e inclinação volar, respectivamente. Não foram encontradas correlações estatisticamente significantes para os parâmetros degrau articular e desvio articular, devido à homogeneidade dos dados. De acordo com a análise das Tabelas 4, 5 e 6 os únicos parâmetros que apresentaram correlação estatisticamente significante foram a inclinação radial e o desvio ulnar. A análise de regressão linear (Figura 2) demonstrou correlação entre ambas as variáveis.

Tabela 4. Valores do coeficiente de correlação de Sperman e p-crítico, encontrados para o parâmetro altura radial correlacionado a parâmetros clínicos

\begin{tabular}{l|c|c}
\hline & $\begin{array}{c}\text { Coeficiente de correlação } \\
\text { de Spearman }\end{array}$ & $\boldsymbol{p}$ \\
\hline Flexão de punho & 0,5991 & 0,13288 \\
\hline Extensão de punho & $-0,0017$ & 0,9946 \\
\hline Desvio radial & 0,0539 & 0,8315 \\
\hline Desvio ulnar & $-0,0127$ & 0,9600 \\
\hline Supinação & 0,3265 & 0,1859 \\
\hline Pronação & 0,0177 & 0,9443 \\
\hline Pinça polpa-polpa & 0,1489 & 0,5554 \\
\hline Pinça trípode & 0,1327 & 0,5996 \\
\hline Pinça chave & 0,0045 & 0,9856 \\
\hline Preensão & 0,0188 & 0,9408 \\
\hline PRWE & 0,0188 & 0,9407 \\
\hline
\end{tabular}

Fonte: Dados da Pesquisa
Tabela 5. Valores do coeficiente de correlação de Sperman e p-crítico, encontrados para o parâmetro inclinação radial correlacionado a parâmetros clínicos

\begin{tabular}{l|c|c}
\hline $\begin{array}{c}\text { Medida correlacionada } \\
\text { com a inclinação radial }\end{array}$ & $\begin{array}{c}\text { Coeficiente de correlação } \\
\text { de Spearman }\end{array}$ & $\boldsymbol{p}$ \\
\hline Flexão de punho & 0,4141 & 0,08754 \\
\hline Extensão de punho & 0,2767 & 0,2663 \\
\hline Desvio radial & 0,2232 & 0,3732 \\
\hline Desvio ulnar & 0,3310 & $0,1797^{*}$ \\
\hline Supinação & 0,5653 & 0,0144 \\
\hline Pronação & 0,0950 & 0,7076 \\
\hline Pinça polpa-polpa & 0,1708 & 0,4979 \\
\hline Pinça trípode & 0,2923 & 0,2391 \\
\hline Pinça chave & 0,0068 & 0,9784 \\
\hline Preensão & $-0,0094$ & 0,9702 \\
\hline PRWE & $-0,0755$ & 0,7657 \\
\hline Fonte: Dados da Pesquisa & &
\end{tabular}

Tabela 6. Valores do coeficiente de correlação de Sperman e p-crítico, encontrados para o parâmetro inclinação volar correlacionado a parâmetros clínicos

\begin{tabular}{l|c|c}
\hline $\begin{array}{c}\text { Medida correlacionada } \\
\text { com a altura radial }\end{array}$ & $\begin{array}{c}\text { Coeficiente de correlação } \\
\text { de Spearman }\end{array}$ & $\boldsymbol{p}$ \\
\hline Flexão de punho & 0,2758 & 0,2679 \\
\hline Extensão de punho & $-0,1465$ & 0,5616 \\
\hline Desvio radial & $-0,0228$ & 0,9283 \\
\hline Desvio ulnar & $-0,0121$ & 0,9619 \\
\hline Supinação & $-0,2336$ & 0,3508 \\
\hline Pronação & $-0,0257$ & 0,9192 \\
\hline Pinça polpa-polpa & 0,0119 & 0,9625 \\
\hline Pinça trípode & 0,0130 & 0,9591 \\
\hline (?) Chave & $-0,3335$ & 0,1762 \\
\hline Preensão & $-0,1968$ & 0,4336 \\
\hline PRWE & $-0,2584$ & 0,3005 \\
\hline Fonte: Dados da Pesquisa & &
\end{tabular}

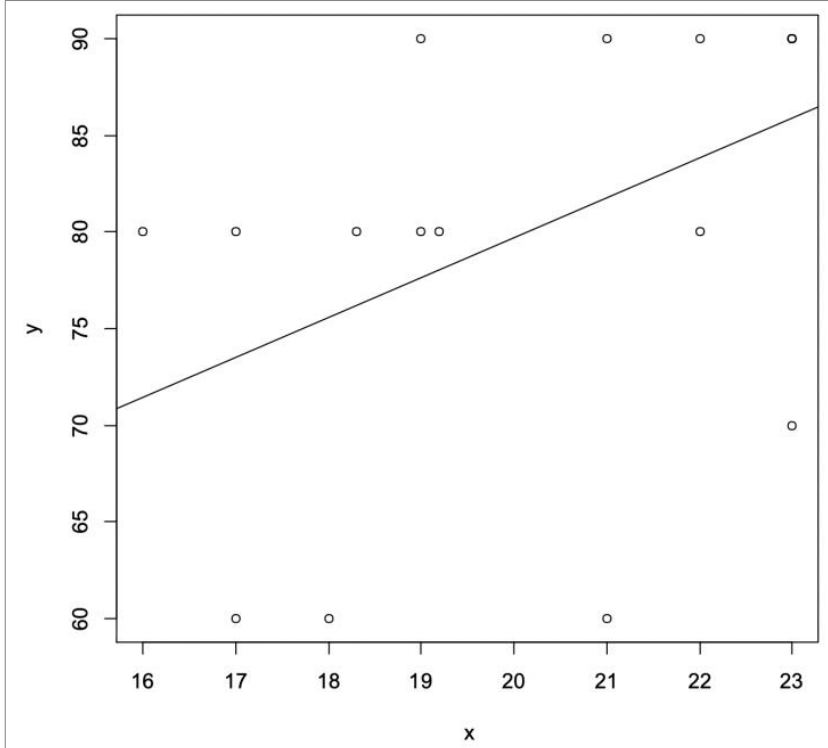

Figura 1: Regressão linear entre a inclinação radial (x) e o desvio ulnar (y) (Fonte: Dados da Pesquisa).

\section{DISCUSSÃO}

O tratamento cirúrgico das fraturas da extremidade distal do rádio é realizado com o objetivo de reduzir adequadamente a fratura, com retorno aos padrões radiológicos normais para o rádio distal, o que possivelmente levaria a um bom resultado funcional ${ }^{25}$. Contudo, este cenário nem sempre é atingido devido diversos fatores como presença de cominuição articular importante, perda óssea, limitação técnica do cirurgião, falha na reabilitação, entre outros. A correlação entre os parâmetros radiográficos pós-operatórios e resultado funcional é amplamente debatido na literatura ${ }^{24,26}$.

Karnezis et al. ${ }^{22}$ demonstraram relação entre encurtamento radial e inclinação volar com o PRWE, 
porém não houve relação entre parâmetros radiográficos com amplitude de movimento e força de preensão.

No estudo de Xavier et al. ${ }^{21}$ observou-se que as medidas radiográficas do lado acometido foram de: $85,4 \%$ do valor do lado não acometido para altura radial, $84 \%$ do valor do lado não acometido para inclinação radial e $86,8 \%$ do valor do lado não acometido para inclinação volar. Não houve correlação entre os parâmetros radiográficos e o escore DASH (Disabilites of the Arm, Shoulder and Hand), questionário subjetivo semelhante ao PRWE.

$\mathrm{O}$ valor médio de PRWE encontrado neste presente estudo foi de 26,16 (DP=32,43), se aproximando do valor de 8,2 encontrado em estudo de Paranaíba et al, que também evidenciou que que não há correlação significativa entre resultado radiográfico e PRWE, situação semelhante encontrada no estudo de Kasapinova et al. ${ }^{26}$. Neste último, salienta-se que a reabilitação e avaliação funcional dos pacientes com fratura da extremidade distal do rádio deve se estender além das deficiências radiográficas e físicas ${ }^{27}$.

Em estudo de Trumble et al. ${ }^{24}$ a média de amplitude de movimento e força de preensão observada no lado operado foi de 75 e 69\% respectivamente em relação ao contralateral, semelhante aos valores de 82,6 e 70,5\% do presente estudo. O degrau e desvio articular e o encurtamento radial apresentaram correlação estatística significativa com o resultado funcional, contudo a inclinação radial e volar não demonstraram esta correlação.

\section{CONCLUSÃO}

As correlações entre os parâmetros radiográficos e os resultados funcionais são difíceis de estabelecer. Em nosso estudo, a inclinação radial e o desvio ulnar apresentam relação com os resultados funcionais encontrados. Porém, novos estudos com amostra maior são necessários para avaliar a real correlação entre as alterações radiográficas e 0 resultado funcional.

\section{REFERÊNCIAS}

1. Court-Brown CM, Caesar B. Epidemiology of adult fractures: a review. Injury, Int. J. Care Injured. 2006;37:691-97.

2. Wolf WS. Distal Radius Fracture. In: Green's Operative Hand Surgery $7^{\text {th }}$ ed. Philadelphia, PA: Elsevier.

3. Alluri RK, Hill JR, Ghiassi A. Distal Radius Fractures: Approaches, Indications, and Techniques. J Hand Surg Am. 2016;41(8):845-54.

4. McQueen MM. Fractures oh the distal radius and ulna. In: Rockwood and Green's fractures in adults $8^{\text {th }}$ ed. Philadelphia, PA: Wolters Kluwer Health.

5. Brogren E, Petranek M, Atroshi I. Incidence and characteristics of distal radius fractures in a southern Swedish region. BMC Musculoskeletal Disorders. 2007;8:48.

6. Flinkkilä T, Sirniö K, Hippi M, et al. Epidemiology and seasonal variation of distal radius fractures in Oulu, Finland. Osteoporos Int. 2011;22(8):2307-12.

7. Róbertsson GO, Jónsson GT, Sigurjónsson K. Epidemiology of distal radius fractures in Iceland in 1985. Acta Orthop Scand 1990;61(5):457-59

8. Sigurdardottir K, Halldorsson S, Robertsson J. Epidemiology and treatment of distal radius fractures in Reykjavik, Iceland, in 2004. Comparison with an Icelandic study from 1985. Acta Orthopaedica 2011; 82(4):494-98.

9. Meena S, Sharma $P$, Sambharia AK, et al. Fractures of distal radius: an overview. J Family Med Prim Care. 2014;3(4):325-32.

10. Metz VM, Gilula LA. Imaging techniques for distal radius fractures and related injuries. Orthop Clin North Am. 1993;24(2):217-28.

11. Reis FB, Faloppa F, Saone RP, et al. Fraturas do terço distal do rádio: classificação e tratamento. Rev Bras Ortop - Vol. 29, N 5 - Maio, 1994.

12. Isani A, Melone CP Jr. Classification and management of intra-articular fractures of the distal radius. Hand Clin. 1988; 4 (3):349-60.

13. Lafontaine M, Hardy D, Delince P. Stability assessment of distal radius fractures. Injury. 1989; 20(4):208-10.

14. Knirk JL, Jupiter JB. Intra-articular fractures of the distal end of the radius in young adult. J Bone Joint Surg Am. 1986;68(5):647-59.

15. Szabo RM, Weber SC. Comminuted intraarticular fractures of the distal radius. Clin Orthop Relat Res. 1988;230:39-48

16. Trumble TE, Culp RW, Hanel DP, et al. Intraarticular fractures of the distal aspect of the radius. Instr Course Lect. 1999;48:465-80.

17. Lipton HA, Wollstein R. Operative treatment of intraarticular distal radial fractures. Clin Orthop Relat Res. 1996;(327):110-24.

18. Bradway JK, Amadio PC, Cooney WP. Open reduction and internal fixation of displaced, comminuted intra-articular fractures of the distal end of the radius. J Bone Joint Surg Am. 1989;71(6):839-47.

19. Melone CP Jr. Distal radius fractures: patterns of articular fragmentation. Orthop Clin North Am. 1993;24(2):239-53.

20. Xavier CRM, Molin DCD, dos Santos RMM, et al. Tratamento cirúrgico das fraturas do rádio distal com placa volar bloqueada: correlação dos resultados clínicos e radiográficos. Rev Bras Ortop. 2011;46(5):505-13.

21. Karnezis IA, Panagiotopoulos E, Tyllianakis M, et al. Correlation between radiological parameters and patient-rated wrist dysfunction following 
fractures of the distal radius. Injury, Int. J. Care Injured.2005;36:1435-39.

22. Schneiders W, Biewener A, Rammelt S, et al. Distal radius fracture. Correlation between radiological and functional results. Unfallchirurg. 2006; 109(10):837-44.

23. Trumble TE, Schmitt SR, Vedder NB. Factors affecting functional outcome of displaced intraarticular distal radius fractures. J Hand Surg Am. 1994;19(2):325-40.

24. Fernandez DL. Should anatomic reduction be pursued in distal radial fractures? J Hand Surg Br. 2000;25(6):523-27.

25. Kasapinova K, Kamiloski V. Outcome evaluation in patients with distal radius fracture. Prilozi. 2011; 32(2):231-46.

26. Paranaíba VF, Santos JBG, Raduan Neto J, Moraes VY, Belotti JC, Faloppa F. Aplicação do PRWE na fratura da extremidade distal do rádio: comparação e correlação dos desfechos consagrados. Rev bras ortop. 2017;52(3):278-83.

27. Rodrigues EKS, Fonseca MCR, MadDermid JC. Brazilian version of the Patient Rated Wrist Evaluation (PRWE-BR): Cross-cultural adaptation, internal consistency, test-retest reliability and constructo validity. J Hand Ther. 2015;28(1):69-76.

\section{CONFLITO DE INTERESSES}

Os autores declaram não haver conflitos de interesse.

\section{AUTOR PARA CORRESPONDÊNCIA}

\section{Breno Alves de Souza Vaz}

Hospital das Clínicas da faculdade de Medicina de Ribeirão Preto da Universidade de São Paulo (USP)

R. Ten. Catão Roxo, 3900 - Vila Monte Alegre, Ribeirão Preto SP, 14015-010

E-mail: breno.a.s.v.@gmail.com.br

Submetido em 19/08/2019

Aceito em 20/04/2020 